\title{
Front motion in an $A+B \rightarrow C$ type reaction-diffusion process: Effects of an electric field
}

\author{
I. Bena, ${ }^{1}$ F. Coppex, ${ }^{1}$ M. Droz, ${ }^{1}$ and Z. Rácz ${ }^{2}$ \\ ${ }^{1}$ Department of Physics, University of Genève, CH-1211 Genève 4, Switzerland \\ ${ }^{2}$ Institute for Theoretical Physics - HAS, Eötvös University, Pázmány sétány 1/a, 1117 Budapest, Hungary
}

(Dated: September 4, 2018)

\begin{abstract}
We study the effects of an external electric field on both the motion of the reaction zone and the spatial distribution of the reaction product, $C$, in an irreversible $A^{-}+B^{+} \rightarrow C$ reaction-diffusion process. The electrolytes $A \equiv\left(A^{+}, A^{-}\right)$and $B \equiv\left(B^{+}, B^{-}\right)$are initially separated in space and the ion-dynamics is described by reaction-diffusion equations obeying local electroneutrality. Without an electric field, the reaction zone moves diffusively leaving behind a constant concentration of $C$-s. In the presence of an electric field which drives the reagents towards the reaction zone, we find that the reaction zone still moves diffusively but with a diffusion coefficient which slightly decreases with increasing field. The important electric field effect is that the concentration of $C$-s is no longer constant but increases linearly in the direction of the motion of the front. The case of an electric field of reversed polarity is also discussed and it is found that the motion of the front has a diffusive, as well as a drift component. The concentration of $C$-s decreases in the direction of the motion of the front, up to the complete extinction of the reaction. Possible applications of the above results to the understanding of the formation of Liesegang patterns in an electric field is briefly outlined.
\end{abstract}

PACS numbers: 05.60.Gg, 64.60.Ht, 75.10.Jm, 72.25.-b

\section{INTRODUCTION}

Nonequilibrium systems exhibiting pattern formation are ubiquitous in nature and these patterns often emerge in the wake of a moving reaction front [1]. A classical example which motivated our present study is the emergence of Liesegang bands 2, 3] associated with the generic reaction scheme $A+B \rightarrow C$ and the subsequent precipitation of $C$-s. In a typical experimental setup, a chemical reactant, $B$ (called inner electrolyte), is disolved in a gel matrix, while a second reactant, $A$ (outer electrolyte), of much higher concentration is brought into contact with the gel. The outer electrolyte diffuses into the gel, reacts with the inner electrolyte, and the reaction front moves into the gel. Under appropriate conditions, the reaction product precipitates and, depending on the geometry of the experimental setup, one can observe the emergence of families of bands or rings perpendicular to the direction of motion of the front [3, 4].

This pattern formation process is a rather complex one, due to the delicate interplay between the motion of the reaction front and the precipitation dynamics of the reaction product $C$. Its study has a history of more than a century [2] and the generic, empirical laws describing it are well established 5]. There is still disagreement, however, about the mechanisms underlying this patternforming process (see [6, 7] for a comparative analysis of the existing theories). Moreover, with the exception of the recently-proposed phase separation (or spinodal decomposition) scenario $[\underline{8},[9]$, the existing theories are essentially qualitative, as they contain parameters that cannot be inferred from experiments, and therefore they do not allow for quantitative predictions or for any direct comparison with the existing experimental data. Having this state of affairs, we believe that it is important to test the spinodal decomposition theory in as many ways as possible.

The basic ingredients of the spinodal decomposition approach are the presence of a moving reaction front and the phase separation that takes place in the wake of the front. The dynamics of the inert reaction product $C$ is assumed to have no feedback on the dynamics of the reagents 10], thus one represents the dynamics as a two-stage process. Namely, (i) the production of $C$ in the moving reaction front is described by reactiondiffusion equations, while (ii) the diffusion and precipitation of $C$ particles is modelled as phase separation (spinodal decomposition) 11] using the Cahn-Hilliard equation [12,13, 14], with a source term corresponding to the production of $C$ by stage (i). All the parameters involved in this model (including the parameters of the LandauGinzburg free energy associated with the dynamics of $C$ particles) are either directly accessible experimentally or can be inferred from experimental data (see [5]).

The first stage $(A+B \rightarrow C)$ of the process appears in many other physical and chemical processes and has been much studied. The dynamics of the front and the spatial distribution of the rate of the production of $C$ (the source for the second stage) are known [15] for the case of neutral reagents $A$ and $B$. It should be realized, however, that $A$ and $B$ are usually electrolytes which dissociate,

$$
A \rightarrow A^{+}+A^{-}, \quad B \rightarrow B^{+}+B^{-},
$$

and the basic reaction process takes place between the 'active' ions, e.g.,

$$
A^{-}+B^{+} \rightarrow C,
$$

while the 'background' ions $A^{+}$and $B^{-}$are not reacting. For the first sight, the background ions may be not important. They just ensure local electroneutrality and the 
Debye screening length is much smaller than the spatial lengths involved in the macroscopic pattern formation. However, as it was shown recently [16], the dynamics of the background ions may generate macroscopic effects (even if the screening length is negligible) and may influence both the propagation of the reaction front and the structure of the resulting pattern. Thus, an obvious way to obtain more insight into the details of the dynamics of Liesegang pattern formation (with the ultimate goal of validating one theoretical scenario or another) is to switch on an electric field and to study its effects.

Experimental and theoretical investigations in this direction have been going on for quite a while, see 17, 18, $19,20,21,22,23,24,25,26,27,28,29$, , 30]. The emerging picture, however, might be sometimes confusing. For example, on the experimental side, Das et al.'s experiments 22, 23] show a diffusive motion of the front for a polarity of the applied field that favors the reaction, and for a reverse polarity, as shown by Sultan et al. 27] the motion of the front acquires a supplementary drift component. These results are just the opposite of those obtained by Lagzi 29], however it can be argued 31] that in the latter case the properties of the intermediate compounds are responsible for this 'anomalous' behavior. As far as the theories are concerned, a drift of the front is obtained analytically in e.g. 20] under the irrealistic assumption of the constancy of the electric field along the system, or it is put 'by hand' as e.g. in [30].

In order to clarify the effect of the electric field on Liesegang patterns, we propose to revisit the problem in the framework of the spinodal decomposition theory. In the present paper, we shall concentrate on the first stage of this scenario, namely on the effect of the electric field on the motion of the reaction front and on the concentration of the reaction product $C$ left behind the front. As mentioned above, the problem of the first stage is more general and thus we feel that the results are of interest in their own. Although we shall mention the implications of the reaction zone results for Liesegang patterns, the calculations and discussions of the second stage of the process will be presented in a forthcoming study.

As it will be discussed in the next section (Sec. III with some details in Appendix A), the new element in our model is that the dynamics of all the ions $\left(A^{+}, A^{-}, B^{+}, B^{-}\right)$is followed by using reaction-diffusion equations obeying the electroneutrality condition. Furthermore, the electric field is taken into account by prescribing a given potential drop between the two ends of the sample, as in real experimental situations. The equations are solved numerically in Sec. [II] where, in view of the possible comparison of our results with experimental data, we concentrate our study on the experimentally relevant range of parameters and observation times.

Our results can be summarized by contrasting them with the fieldless case where the reaction front moves diffusively, and it leaves behind a constant concentration $c_{0}$ of the reaction product $C$ 15, 16. In the presence of the electric field, the polarity of the field is an im- portant factor. If the field favours the reaction (in the sense that it drives the reacting ions $A^{-}$and $B^{+}$towards the reaction zone), then the motion of the reaction front remains diffusive, and the concentration of the reaction product $c(x)$ increases linearly in space in the direction of the motion of the front. The diffusive front motion is in agreement with Das et al.'s experiments [22, 23] and in contrast with theoretical conjectures about constantvelocity drifts [20, 30]. An important remark is that this case is difficult to handle, since a strong electric field develops in the reaction zone (especially for large applied tensions), which leads to numerical instabilities and thus restricts our numerical solutions to relatively short times.

In the case of opposite polarity of the applied field, the motion of the reaction zone is still diffusive at short times, but a crossover to drift at large times, as seen in Sultan's experiments [27], can be inferred from numerical data. As far as the concentration of the reaction product is concerned, we find that at small times it decreases linearly in the direction of the motion of the front, while a slow-down of this decrease is noticed for larger times, up to the extinction of the reaction.

The conclusions and the implications of the results for Liesegang phenomena will be presented in Sec. IV. Finally, some salient details of the problem such as the electric-field profiles, the origin of the numerical instabilities, the time evolution of the electric current, the finite-size effects, etc. are discussed in Appendix B.

\section{THE MODEL IN THE PRESENCE OF AN APPLIED ELECTRIC FIELD}

The experimental setup we shall consider is represented schematically in Fig. 11 The two electrolytes are initially separated from each other, each is uniformly spread into a gel matrix, and the concentration of $A$ 's is much larger than that of $B$ 's. Thus the reaction front $A^{-}+B^{+} \rightarrow C$ moves to the right along the column. An appropriate choice of the experimental conditions (type of reagents, concentrations, etc.) leads to quasiperiodic precipitation of the inert reaction product $C$ in the wake of the moving front - the Liesegang patterns, see Fig. [1] Due to the presence of the gel the convection phenomena are absent, and thus the evolution of the concentration fields of the particles is accurately described by reactiondiffusion equations.

Our model is based on several simplifying assumptions, and we refer the reader to Appendix A for more details on a more general model.

(i) The first assumption is that the system is onedimensional, which means that all the relevant quantities depend on a single spatial coordinate $x$, with $-L_{A} \leq x \leq$ $L_{B}$ being the spatial extent of the system. This is essentially realized in practice provided that the length of the gel column is much larger than its width. The edge effects in the transverse directions might have some slight consequences (e.g., as reported in 22], in the presence of 


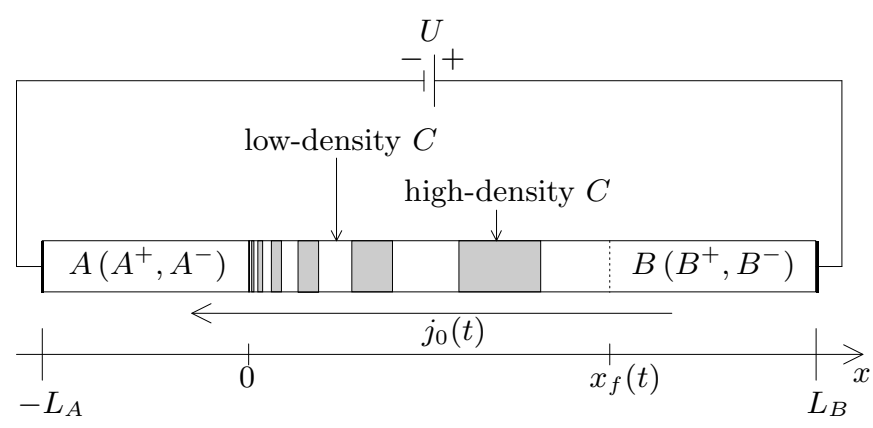

FIG. 1: Schematic representation of the system under study. The electrolytes $A\left(A^{+}, A^{-}\right)$and $B\left(B^{+}, B^{-}\right)$are initially located in the regions $\left[-L_{A}, 0\right)$, respectively $\left(0, L_{B}\right]$. The precipitation bands, i.e., the alternation of high-density- $C$ regions (shaded areas) and low-density- $C$ ones, emerge in the wake of the moving reaction front (dashed line at $x_{f}(t)$ ). For an applied tension $U$, an electric current of density $j_{0}(t)$ is flowing through the system.

an external electric field the diameter of the tube has a small influence on the propagation of the reaction front). However, the final pattern is still one-dimensional to a very good accuracy.

(ii) We consider instantaneous $100 \%$ dissociation of the electrolytes $A$ and $B$ according to Eq. (1) (the assumption of "ideally strong" acid and basis). Correspondingly, only $A^{ \pm}$and $B^{ \pm}$ions (and the reaction product $C$ ) are present in the system.

(iii) The ions $A^{-}$and $B^{+}$are reacting irreversibly with an infinite reaction rate. This can be justified by the fact that the characteristic time of the reaction process is 3 to 6 orders of magnitude less than the time scales invloved in the diffusion and precipitation processes, see, e.g., [7, 16]. In this case, one has a pointlike reaction front at a time-dependent position $x_{f}(t)$ 32].

(iv) The electroneutrality approximation 33 - according to which the local charge density is zero on space scales that are relevant to pattern formation - reads

$$
\sum_{i} z_{i} n_{i}(x, t)=0,
$$

where $z_{i}$ is the charge of the $i$-th ion - in terms of the elementary charge $q$ - and $n_{i}(x, t)$ denotes its density. As discussed in detail in [16, 34], this condition is well fulfilled in the systems we are investigating. Moreover, we shall consider monovalent ions, $\left|z_{i}\right|=1$ for all $i$.

(v) Finally, we consider equal diffusion coefficients of the ions, $D_{i}=D$ for all $i$.

The initial conditions for the concentration profiles $a^{ \pm}(x, t)$ and $b^{ \pm}(x, t)$ of the $A^{ \pm}$and $B^{ \pm}$ions correspond to initially separated reagents,

$$
\begin{aligned}
& a^{-}(x, t=0)=a^{+}(x, t=0)=a_{0}, \\
& b^{+}(x, t=0)=b^{-}(x, t=0)=0 \\
& \quad \text { for }-L_{A} \leq x \leq 0 \\
& a^{-}(x, t=0)=a^{+}(x, t=0)=0, \\
& b^{+}(x, t=0)=b^{-}(x, t=0)=b_{0}, \\
& \text { for } 0<x \leq L_{B},
\end{aligned}
$$

with $a_{0} \gg b_{0}$. We also suppose that the concentrations of the ions are maintained fixed at the borders,

$$
\begin{aligned}
& a^{+}\left(-L_{A}, t\right)=a^{-}\left(-L_{A}, t\right)=a_{0}, \\
& b^{+}\left(-L_{A}, t\right)=b^{-}\left(-L_{A}, t\right)=0, \\
& a^{+}\left(L_{B}, t\right)=a^{-}\left(L_{B}, t\right)=0, \\
& b^{+}\left(L_{B}, t\right)=b^{-}\left(L_{B}, t\right)=b_{0},
\end{aligned}
$$

i.e., the system is in contact at its left and right borders with two 'infinite' reservoirs of ions $A^{ \pm}$and $B^{ \pm}$, respectively.

Under these assumptions, the general evolution equations for the concentration profiles $a^{ \pm}(x, t)$ and $b^{ \pm}(x, t)$ of the $A^{ \pm}$and $B^{ \pm}$ions presented in the Appendix A acquire the following simplified expressions:

(i) For $x<x_{f}(t)$ (i.e., in the wake of the reaction front)

$$
\begin{aligned}
& \frac{\partial a^{-}(x, t)}{\partial t}=D \frac{\partial^{2} a^{-}}{\partial x^{2}}+\frac{j_{0}}{2 q} \frac{\partial}{\partial x}\left(\frac{a^{-}}{a^{+}}\right), \\
& b^{+}(x, t) \equiv 0, \\
& \frac{\partial a^{+}(x, t)}{\partial t}=D \frac{\partial^{2} a^{+}}{\partial x^{2}}, \\
& \frac{\partial b^{-}(x, t)}{\partial t}=D \frac{\partial^{2} b^{-}}{\partial x^{2}}+\frac{j_{0}}{2 q} \frac{\partial}{\partial x}\left(\frac{b^{-}}{a^{+}}\right) .
\end{aligned}
$$

(ii) For $x>x_{f}(t)$ (i.e., ahead the reaction front)

$$
\begin{aligned}
& a^{-}(x, t) \equiv 0, \\
& \frac{\partial b^{+}(x, t)}{\partial t}=D \frac{\partial^{2} b^{+}}{\partial x^{2}}-\frac{j_{0}}{2 q} \frac{\partial}{\partial x}\left(\frac{b^{+}}{b^{-}}\right), \\
& \frac{\partial a^{+}(x, t)}{\partial t}=D \frac{\partial^{2} a^{+}}{\partial x^{2}}-\frac{j_{0}}{2 q} \frac{\partial}{\partial x}\left(\frac{a^{+}}{b^{-}}\right), \\
& \frac{\partial b^{-}(x, t)}{\partial t}=D \frac{\partial^{2} b^{-}}{\partial x^{2}} .
\end{aligned}
$$

Here $j_{0}$ is the time-dependent electric current density

$$
j_{0}(t)=\frac{-2 q F D U}{R T\left(\int_{-L_{A}}^{x_{f}(t)} \frac{d x}{a^{+}}+\int_{x_{f}(t)}^{L_{B}} \frac{d x}{b^{-}}\right)}
$$

determined by the constant voltage difference $U=$ $V\left(L_{B}\right)-V\left(-L_{A}\right)$ applied between the two ends of the system [35]. $F=q N_{A}$ is Faraday's constant (i.e., the electric charge transported by a mole of monovalent positive ions), $R$ is the universal gas constant, while $T$ is the 
temperature. Finally, the local electric field is given by:

$$
\begin{aligned}
E(x, t) & =\frac{j_{0}(t)}{\frac{q F D}{R T} \sum_{i} n_{i}(x, t)} \\
& =\frac{-2 U}{\sum_{i} n_{i}\left(\int_{-L_{A}}^{x_{f}(t)} \frac{d x}{a^{+}}+\int_{x_{f}(t)}^{L_{B}} \frac{d x}{b^{-}}\right)} .
\end{aligned}
$$

At a first sight, one would be tempted to conclude that the equations (6)-(13) for the concentration fields look simple, but actually (i) they are nonlinearly coupled through the integral quantity $j_{0}(t)$, according to Eq. (14), and (ii) they acquire time-dependent boundary conditions,

$$
\begin{aligned}
& a^{-}\left(x_{f}(t)\right)=b^{+}\left(x_{f}(t)\right)=0, \\
& \left|j_{a^{-}}\left(x_{f}(t)\right)\right|=\left|j_{b^{+}}\left(x_{f}(t)\right)\right| .
\end{aligned}
$$

which are complicated to handle. The meaning of these latter conditions is just that the concentrations of the reagents are zero at the front, and their flux towards the reaction front are equal.

Even under the simplifying assumption presented above, an analytic solution for the concentration profiles of the ions and thus for the motion of the front seems hopeless. We thus turned to numerical solutions, whose main results are presented in the following Section.

\section{NUMERICAL RESULTS}

As already mentioned in the Introduction, we decided to focus our analysis on situations that are experimentally relevant. In particular, although we are aware of different other regimes that might appear, we restrict the presentation to the ones that are most likely to appear in experiments. The choice of the parameters intends thus to mimic real experimental situations, namely, we considered concentrations of the ions $a_{0}$ and $b_{0}$ of the order $10^{-3} \mathrm{M}-10 \mathrm{M}$, lengths of the system $L=L_{A}+L_{B}$ of some tenths centimeters, and tensions $U$ applied between system's edges such that the corresponding field intensity $U / L$ varies between $\pm 15 \mathrm{~V} / \mathrm{m}$. The common diffusion coefficient of the ions was chosen as $D=10^{-9}$ $\mathrm{m}^{2} / \mathrm{s}$ for all the calculations. Finally, times of observation of the system that vary between a few hours and some tenth days. Note that this observation time corresponds to the real experimental time and not to the time required by the numerical calculations. As far as the actual numerical calculations are concerned, we integrated the partial differential equations (6) -(13) using a fourth-order Runge-Kutta integration scheme in time and a first-order differenciation procedure in space.

We shall generally present the results that were obtained for a polarity $U>0$ of the applied field that favors the reaction, i.e., that drives the $A^{-}$and $B^{+}$ions towards the reaction zone. This is the case that is apriori more interesting for the formation of the Liesegang pattern. However, this situation is numerically difficult, since a strong electric field develops rapidly (especially for large applied tensions) in the reaction zone, thus leading to the appearance of numerical instabilities which limit the observation time. For more details on this point see Appendix B.

We also indicate the main results for the opposite polarity of the electric field, for which the production of $C$ is slowed down and finally stopped. This is a situation that becomes rather rapidly uninteresting from the point of view of the pattern formation.

\section{A. Front propagation}

In the limit of an infinite reaction rate, the reaction zone reduces to a point whose position $x_{f}(t)$ is moving to the right in the experimental setup of Fig. 1]

The main result of the simulations we carried on for the case of the field favoring the reaction is that the motion of the front is diffusive with an effective diffusion coefficient $D_{f}$,

$$
x_{f}(t)=\sqrt{2 D_{f} t} .
$$

The above statement is valid as far as the border effects are negligible (see below) and for the parameter domains that are experimentally relevant. This result is in agreement with the rather accurate experimental results of Das et al. [22, 23], but contradicts some previous theoretical considerations [20, 30] which, however, in our opinion, have no justification.

Figure 2 shows the trajectory of the reaction front for various tensions $U / L$ applied to the system. A first remark would be that the effect of the electric field on front's motion is very small. One is also confronted with a counterintuitive result (that has also been seen experimentally 22,23$]$ ), namely that the motion of the front is very slightly slowed down with increasing field (although the polarity of this field was chosen to favor the reaction). We had to limit the observation times for these large tensions to 5 hours, because of the numerical instabilities already invoked above. Of course, for smaller tensions one can go to longer observation times, like e.g., in Figs. 4 and 5

A look at the concentration profiles of the ions, see Fig. 3. allows to sketch an intuitive explanation of this unexpected behavior. Namely, if no reaction takes place, in the presence of an electric field with a polarity $U>0$ the negative $A^{-}$ions will be pushed ahead the positive ones (and the corresponding symmetric situation for the $B^{ \pm}$ions), as it can be seen from the first panel of the figure. With an infinite-rate reaction present, see the second panel of the figure, the $A^{-}$ions are consumed at the front and the $A^{+}$ions are forced to move ahead, against the electric field (a similar, but symmetric situation occurs for the $B^{ \pm}$ions). Therefore, the motion should be 


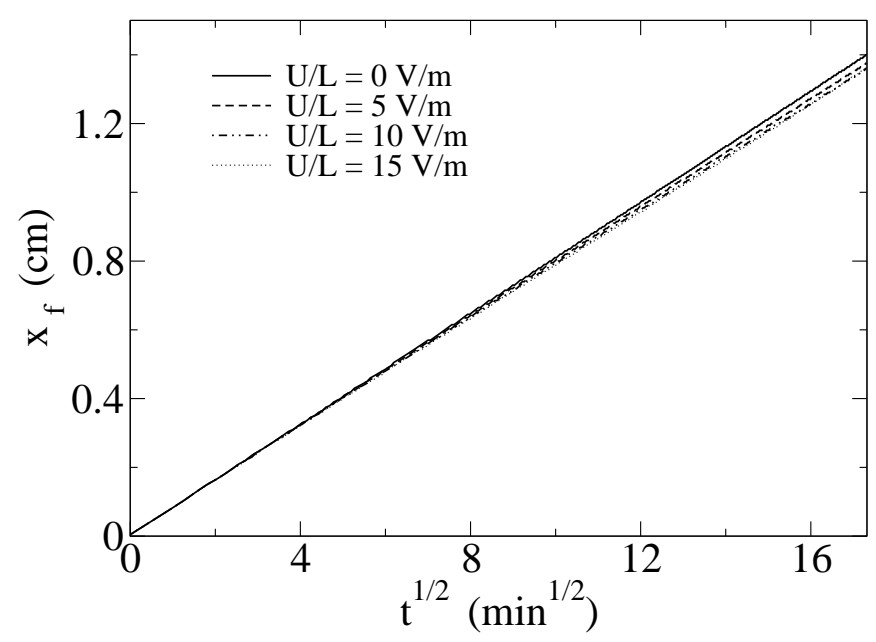

FIG. 2: Position of the reaction front as a function of the square root of time for different values of the intensity $U / L$ of the applied field. The values of the other parameters are: $L_{A}=1 \mathrm{~cm}, L_{B}=20 \mathrm{~cm}, a_{0}=10 \mathrm{M}$, and $b_{0}=0.1 \mathrm{M}$. The observation time is limited to 5 hours (see the main text).

diffusive and has to slow down slightly with increasing field.
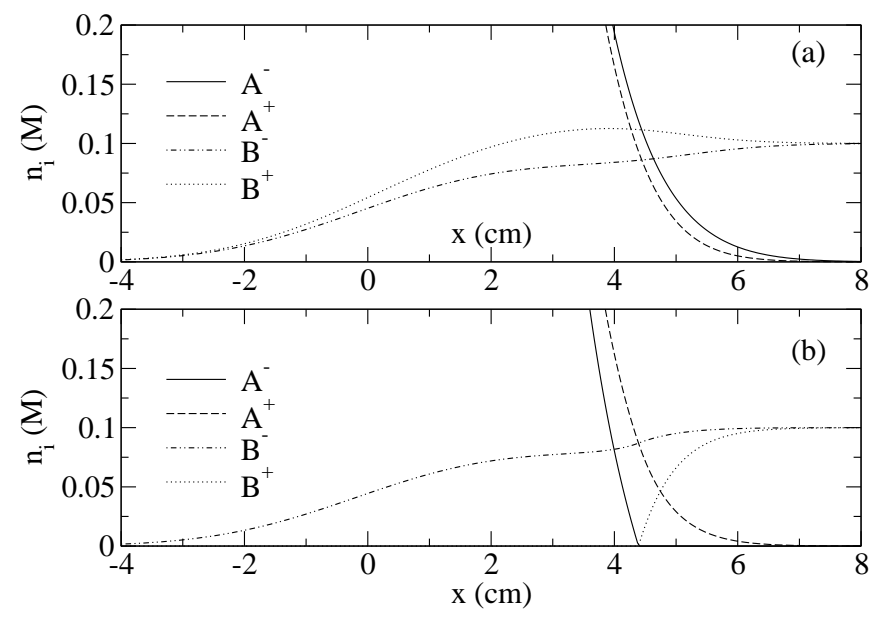

FIG. 3: Two snapshots of the concentration profiles of the ions in the presence of an applied electric field $U / L=0.5$ $\mathrm{V} / \mathrm{m}$ : (a) without reaction (b) with infinite reaction rate. The observation time is of 50 hours and the values of the other parameters are $L_{A}=L_{B}=100 \mathrm{~cm}, a_{0}=10 \mathrm{M}$, and $b_{0}=0.1 \mathrm{M}$.

However, for all the practical purposes (e.g., the pro- duction of $C$ ) one can neglect the dependence of the diffusion coefficient $D_{f}$ on the applied tension and consider its fieldless expression given by [8]:

$$
\operatorname{erf}\left(\sqrt{\frac{D_{f}}{2 D}}\right)=\frac{\left(a_{0} / b_{0}\right)-1}{\left(a_{0} / b_{0}\right)+1},
$$

that corresponds to an increase in $D_{f}$ with increasing concentrations ratio $\left(a_{0} / b_{0}\right)$, see Fig. 4. A qualitative comparison of Figs. 2 and 4 shows that $D_{f}$ is much more sensitive to changes in the concentration ratio $\left(a_{0} / b_{0}\right)$ than to changes in the applied field $U / L$.

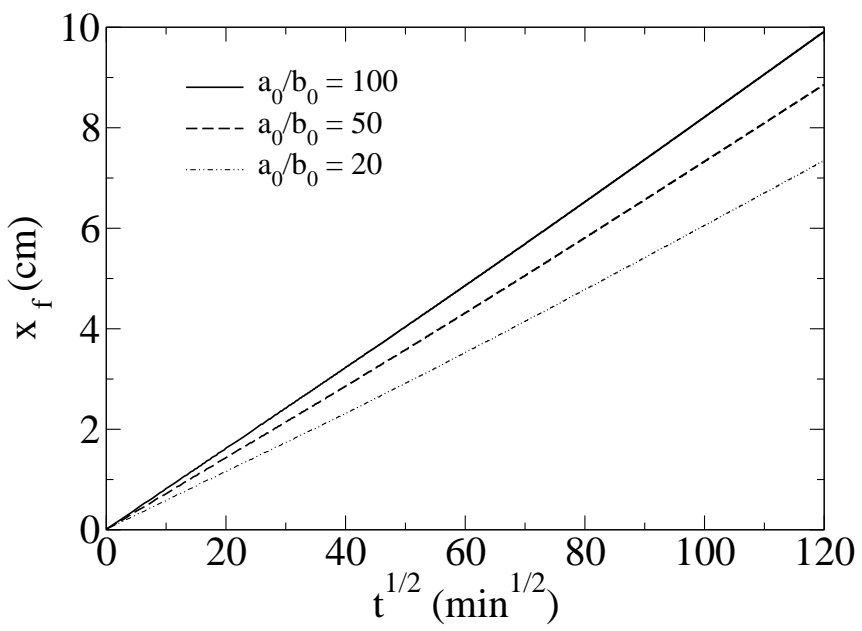

FIG. 4: Position of the reaction front as a function of the square root of time for three values of the concentrations ratio $\left(a_{0} / b_{0}\right)$. The values of the other parameters are: $L_{A}=1 \mathrm{~cm}$, $L_{B}=100 \mathrm{~cm}$, and $U / L=0.5 \mathrm{~V} / \mathrm{m}$. The observation time is 10 days.

The finite spatial extent of the system does not affect the diffusive behavior of the front until the reaction front reaches the very vicinity of the borders. Then the front slows down and stops, and there is a rapid accumulation of $C$ product at the border. However, for the usual observation times we are dealing with, this regime of the front is not relevant, see Appendix B for more details.

\section{B. The concentration of the reaction product $C$}

We come now to the most important results from the point of view of the subsequent pattern formation through precipitation of $C$. Namely, the study of the influence of the applied electric field on the production of $C$ in the wake of the moving front.

As well-known (see, e.g., [8, 15, 16]), in the absence of an applied electric field the concentration of $C$ left behind the reaction front is constant, and its value $c_{0}$ is determined by the initial concentrations of the ions $a_{0}$ and $b_{0}$, 
and by their diffusion coefficients. In the particular case of equal diffusion coefficients $D$ of the ions, its value is given by:

$$
c_{0} \approx a_{0} K \sqrt{2 D / D_{f}},
$$

where $K \equiv\left(1+b_{0} / a_{0}\right)(2 \sqrt{\pi})^{-1} \exp \left(-D_{f} / 2 D\right)$, and the diffusion coefficient $D_{f}$ of the front is given by Eq. (18).

When an electric field is applied to the system, the concentration of $C$ left behind the moving reaction front is no longer constant, but becomes space-dependent, $c=$ $c(x)$.

There are two factors that determine the instantaneous quantity of $C$ that is produced in the wake of the front, namely: (i) The velocity of the front, $\dot{x}_{f}=d x_{f}(t) / d t$. As discussed above, this one is diffusive, with a diffusion coefficient $D_{f}$ that is essentially unaffected by the externally applied electric field, $\dot{x}_{f}=\sqrt{D_{f} / 2 t}$.

(ii) The instantaneous value of the current of the reacting ions $A^{-}$and $B^{+}$that arrive at the reaction point, $\left|j_{a^{-}}\left(x_{f}(t)\right)\right|=\left|j_{b^{+}}\left(x_{f}(t)\right)\right|$, that has a diffusive component, as well as a component proportional to the electric current density $j_{0}(t)$.

A first regime, that appears without exception when the polarity of the applied field favors the reaction, is a linear increase of $c$ with $x$, with a slope that is proportional with the applied tension, see Fig. [5 Moreover, the slope is proportional to $c_{0}$, the concentration of $C$ in the absence of the field, given by Eq. (19).

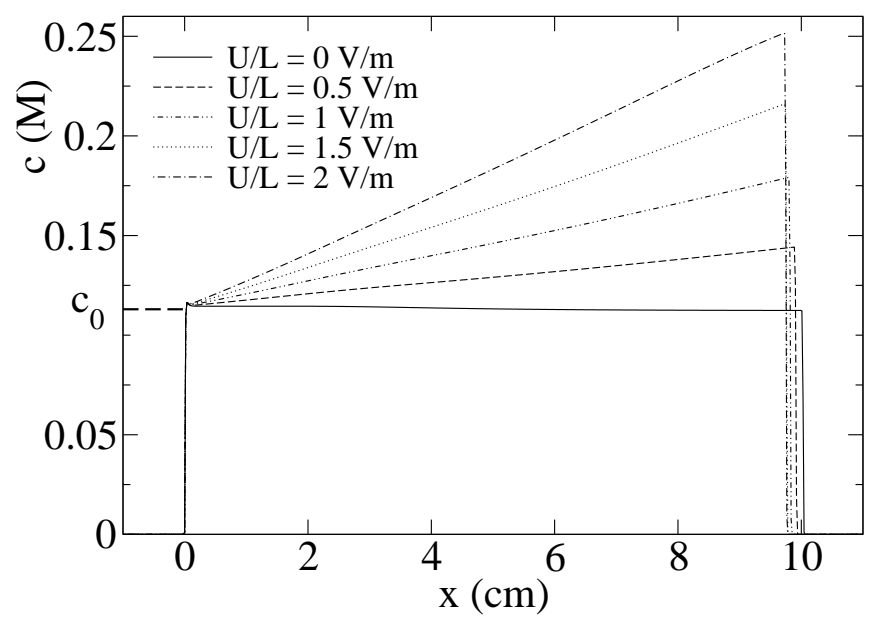

FIG. 5: The density of the reaction product $C$ left behind the reaction front for different values of the electric field $U / L$ applied to the system. The observation time is $t=10$ days. The values of the other parameters are: $L_{A}=1 \mathrm{~cm}, L_{B}=100$ $\mathrm{cm}, a_{0}=10 \mathrm{M}$, and $b_{0}=0.1 \mathrm{M}$.
Thus the numerical results can be summarized in

$$
c(x)=c_{0}\left[1+\left(\alpha_{c} U / L\right) x\right],
$$

and for the profiles in Fig. [5] one inferres $\alpha_{c} \approx 5 V^{-1}$.

This behavior of the production of $C$ can be easily understood once we notice that for the relevant observation times the current density $j_{0}$ is practically constant (see below), and thus $\left|j_{a^{-}, b^{+}}\left(x_{f}\right)\right|$ has a constant $\sim j_{0}$ and a diffusive part $\sim 1 / \sqrt{t}$. This fact, in conjunction with the diffusive motion of the front, leads to a density of $C$ in the wake of the front

$$
c\left(x_{f}\right) \sim\left|j_{a^{-}, b^{+}}\left(x_{f}\right)\right| / \dot{x}_{f} \sim \sqrt{t}+\text { const. } \sim x_{f}+\text { const. },
$$

i.e., a linear increase with $x$.

The regime of constancy of the current density $j_{0}$ is discussed in more details in the Appendix B. It is maintained as long as the total electric resistance of the system is practically constant. This means as long as the resistivity of the depletion (low ion concentrations) region that develops around the moving front remains unimportant as compared to the resistance of the rest 'unperturbed' part of the system. Of course, the longer the system, the longer this regime lasts. For most experimental situations, this is indeed the only observable regime.

\section{Reverse polarity of the applied tension}

We present below the main results for a polarity of the applied tension $U<0$ that does not favour the reaction, i.e., tends to push the $A^{-}$and $B^{+}$ions far apart. A first result is that the motion of the front is no longer purely diffusive, but, as can be seen from Fig. 6] a drift component emerges. The curves can be well fit using the expression

$$
x_{f}(t)=\sqrt{2 D_{f} t}+v_{f} t,
$$

with, however, the drift part playing a significant contribution only for sufficiently long times and/or high tensions, in agreement with experimental data [27].

A naive attempt to justify qualitatively this behavior of the front appeals to the type of argument we have already used in Sec. IIIA for the case of the favorable polarity of $U$ (in order to explain, there, the diffusive motion of the front). Namely, in the case $U<0$, the $A^{+}$ions always move ahead the (reacting) $A^{- \text {'s }}$ (and the symmetric situation for the $B^{ \pm}$ions), as seen in Fig. 7 but in this case their motion is in the sense of the applied field, and might allow for a diffusive, as well as a drift component of front's motion.

The concentration $c$ of the reaction product decreases nonlinearly with $x$, as seen in Fig. 8, faster when the applied field intensity $|U| / L$ is larger, and up to the complete stop of the reaction (when obviously Eq. (22) becomes meaningless). Thus this situation becomes rather rapidly uninteresting from the point of view of Liesegang pattern formation, as it leads to the disappearance of the pattern. 


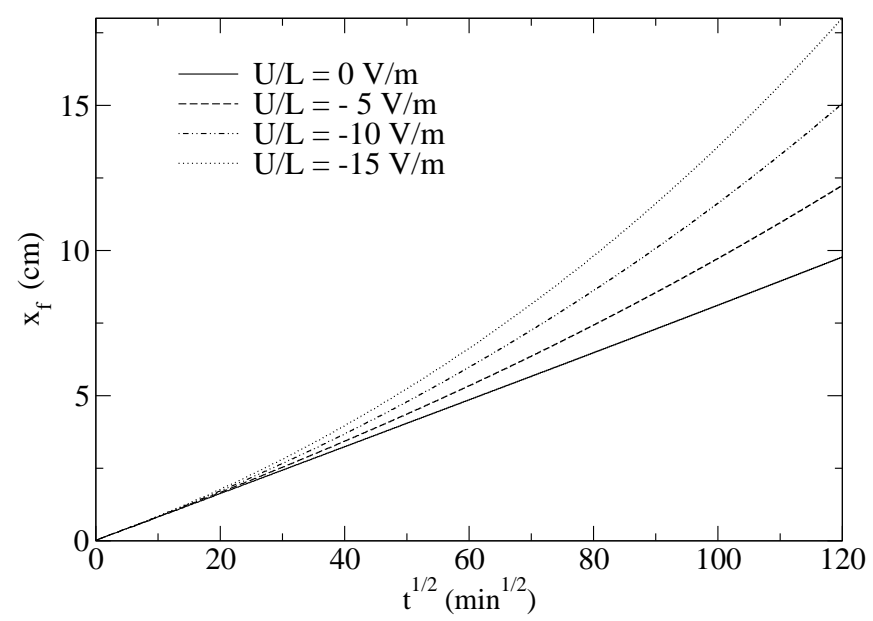

FIG. 6: The position of the reaction front as a function of the square root of time for different values of the applied field $U / L<0$ as compared to the fieldless case. One notices the drift component of the motion. The observation time is $t=10$ days. The values of the other parameters are: $L_{A}=2 \mathrm{~cm}$, $L_{B}=40 \mathrm{~cm}, a_{0}=10 \mathrm{M}$, and $b_{0}=0.1 \mathrm{M}$.
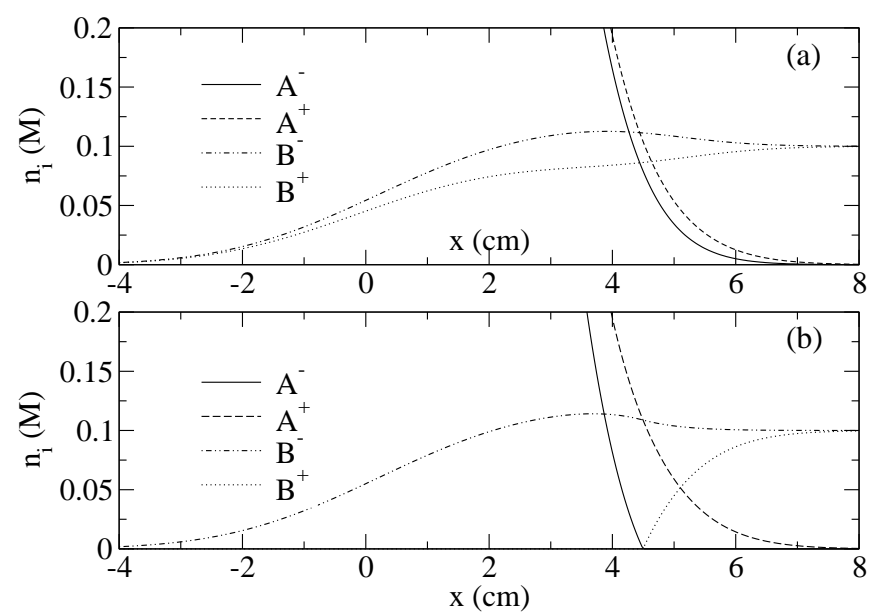

FIG. 7: Two snapshots of the concentration profiles of the ions in the presence of an applied electric field $U / L=-0.5$ $\mathrm{V} / \mathrm{m}$ : (a) without reaction (b) with infinite reaction rate. The observation time is of 50 hours and the values of the other parameters are $L_{A}=L_{B}=100 \mathrm{~cm}, a_{0}=10 \mathrm{M}$, and $b_{0}=0.1$ M.

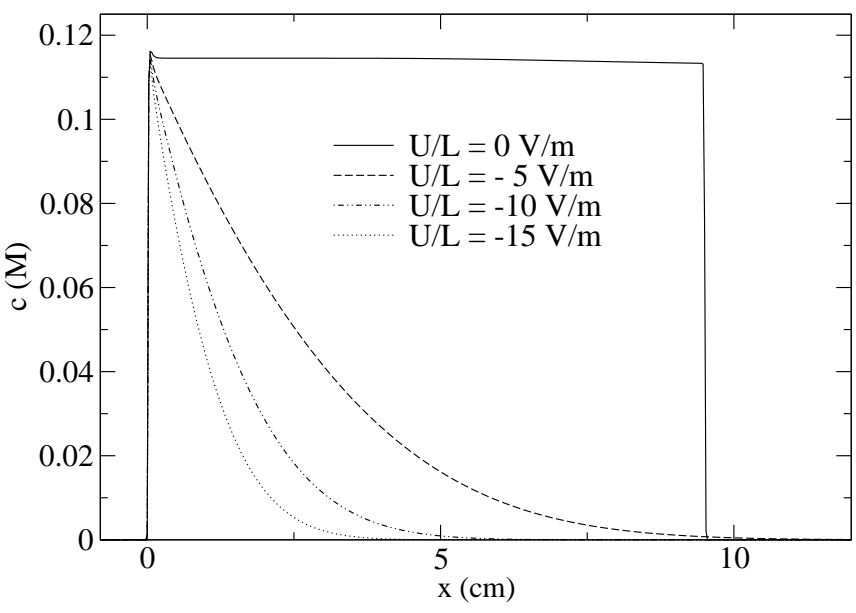

FIG. 8: The density of the reaction product $C$ left behind the reaction front for different values of the applied field $U / L<$ 0 as compared to the fieldless case. One notices the rapid extinction of the reaction with increasing field intensity $|U| / L$. The observation time is $t=10$ days. The values of the other parameters are: $L_{A}=2 \mathrm{~cm}, L_{B}=40 \mathrm{~cm}, a_{0}=10 \mathrm{M}$, and $b_{0}=0.1 \mathrm{M}$.

\section{CONCLUSIONS AND PERSPECTIVES}

In this paper we examined the influence of an applied electric field on the propagation of the reaction front and on the production of $C$ for an $A^{-}+B^{+} \rightarrow C$ reaction in a one-dimensional geometry that is specific to Liesegangpattern experiments. We deliberately concentrated the presentation of our results on the experimentally relevant parameters and observation times (although, for other ranges of the parameters and longer observation times other regimes might come into play). Our main conclusions are that for a polarity of the applied field that favours the chemical reaction, (i) the motion of the reaction front is diffusive, with a diffusion coefficient practically unaffected by the applied field, and (ii) the concentration of the reaction product $c(x)$ left behind the front increses linearly with $x$, with a slope that is proportional to the applied tension.

For the reverse polarity (i.e., that unfavours the reaction), (i) the motion of the front has a diffusive as well as a drift component, and (ii) the concentration $c(x)$ of the reaction product left by the front decreases nonlinearly with $x$ up to the complete stop of the reaction.

The above results on the motion of the reaction front are in agreement with the experiments in 22, 23, 27], but appear to be at variance with the front motion in [29].

The data we presented are based on a set of approximations. Any of them can be relaxed and/or improved, but 
we have evidences that none of them affects essentially our conclusions.

The next step would be to use the above results to determine the influence of the applied tension on the characteristics of the Liesegang patterns. This can be easily done in the framework of the spinodal decomposition scenario. The dynamics of the $C$ particles is still described by a Cahn-Hilliard equation, but this time the source term is modified (as compared to the fieldless case, see [8]) in agreement with our conclusions (i) and (ii) above. A rapid examination of these elements already allows us to predict for a favourable polarity $U>0$ a decrease in the spacing between the bands (the more pronounced the higher the applied tension), and, for sufficiently long times and/or high enough tensions, the disappearance of the pattern. For the reverse polarity $U<0$, the pattern (if any) will present an increase in the spacing between bands as compared to the fieldless case, followed by a rather rapid disappearance of the pattern. A detailed study of this problem will be given in a further publication.

\section{APPENDIX A: THE GENERAL ONE-DIMENSIONAL MODEL}

We present below the general one-dimensional evolution equations for the concentration profiles of the participating species when we do not the approximations of ideally strong acid and basis, infinite reaction rate, and equal diffusion coefficients of the ions (see the main text).

When relaxing the assumption of ideally strong acid and basis for the electrolytes $A$ and $B$, one has to take into account their diffusive motion and their finite dissociation, i.e., the following dynamics of their concentration profiles $a(x, t)$ and $b(x, t)$ :

$$
\begin{aligned}
& \frac{\partial a(x, t)}{\partial t}=D_{a} \frac{\partial^{2} a}{\partial x^{2}}-\lambda_{a}\left(K_{a} a-a^{-} a^{+}\right) \\
& \frac{\partial b(x, t)}{\partial t}=D_{b} \frac{\partial^{2} b}{\partial x^{2}}-\lambda_{b}\left(K_{b} b-b^{-} b^{+}\right) .
\end{aligned}
$$

Here $a^{-}(x, t), a^{+}(x, t), b^{+}(x, t)$, and $b^{-}(x, t)$ are the concentrations of the appropriate ions resulting from $A$ and $B$, respectively, and $\lambda_{a, b}$ are the relaxation constants to the respective dissociation equilibria characterized by the dissociation constants $K_{a}$ and $K_{b}$.

As already mentioned, $A^{+}$and $B^{-}$are not reacting, while the ions $A^{-}$and $B^{+}$are reacting irreversibly with a certain reaction rate $k$, that we shall consider finite,

$$
A^{-}+B^{+} \rightarrow C
$$

We assume that the dynamics of the inert reaction product $C$ has no feedback on the dynamics of the reagents [10].

The macroscopic evolution equations for the concentrations of the ions in the presence of an electric field
$E(x, t)$, by considering different diffusion coeficients of the ions, read:

$$
\begin{aligned}
\frac{\partial a^{-}(x, t)}{\partial t}= & D_{a}^{-} \frac{\partial^{2} a^{-}}{\partial x^{2}}+\lambda_{a}\left(K_{a} a-a^{-} a^{+}\right)-k a^{-} b^{+} \\
& -D_{a}^{-} z_{a}^{-} \frac{F}{R T} \frac{\partial\left(a^{-} E\right)}{\partial x} \\
\frac{\partial b^{+}(x, t)}{\partial t}= & D_{b}^{+} \frac{\partial^{2} b^{+}}{\partial x^{2}}+\lambda_{b}\left(K_{b} b-b^{+} b^{-}\right)-k a^{-} b^{+} \\
& -D_{b}^{+} z_{b}^{+} \frac{F}{R T} \frac{\partial\left(b^{+} E\right)}{\partial x} \\
\frac{\partial a^{+}(x, t)}{\partial t}= & D_{a}^{+} \frac{\partial^{2} a^{+}}{\partial x^{2}}+\lambda_{a}\left(K_{a} a-a^{-} a^{+}\right) \\
& -D_{a}^{+} z_{a}^{+} \frac{F}{R T} \frac{\partial\left(a^{+} E\right)}{\partial x} \\
\frac{\partial b^{-}(x, t)}{\partial t}= & D_{b}^{-} \frac{\partial^{2} b^{-}}{\partial x^{2}}+\lambda_{b}\left(K_{b} b-b^{+} b^{-}\right) \\
& -D_{b}^{-} z_{b}^{-} \frac{F}{R T} \frac{\partial\left(b^{-} E\right)}{\partial x}
\end{aligned}
$$

Here $D_{a, b}^{ \pm}$are the respective diffusion coefficients of the ions, $F=q N_{A}$ is Faraday's constant (i.e., the electric charge transported by a mole of monovalent positive ions), $R$ is the universal gas constant, while $T$ is the temperature. The $z_{i}$-s are signed integers giving the charge of the $i$-th ion, $i=a^{ \pm}, b^{ \pm}$, in units of elementary charge $q$, and we hold the local electroneutrality assumption [33], i.e.,

$$
\sum_{i} z_{i} n_{i}(x, t)=0
$$

at any point and at any time $\left(n_{i}(x, t)\right.$ are the concentrations of the ions). It is useful to recall that indeed local electroneutrality assumption is well-justified for Liesegang type of experiments: at characteristic ion concentrations $\sim 10^{-3}-10 \mathrm{M}$ usually present in Liesegang experiments, the Debye screening length is of the order of $\sim 10^{-10} \mathrm{~m}-10^{-8} \mathrm{~m}$; thus, it is indeed negligible as compared to the other length scales present in the system - lengths of the precipitation zones of the order $\sim 10^{-3}$ $\mathrm{m}$, and width of the reaction zone $\sim 10^{-6} \mathrm{~m}$. See [16, 34] for a more detailed discussion of this point.

As known from textbooks 33], the local electric field $E(x, t)$ in the above equations is determined both by the externally applied field and by the local electroneutrality condition, and it is given by:

$$
E(x, t)=\frac{\frac{j_{0}(t)}{q}+\sum_{i} D_{i} z_{i} \frac{\partial n_{i}}{\partial x}}{\frac{F}{R T} \sum_{i} D_{i} z_{i}^{2} n_{i}} .
$$

$j_{0}(t)$ is the electric current density, flowing through the system. Note that in view of the electroneutrality condition $j_{0}$ is divergence-free, i.e., for the one-dimensional case it is only time-dependent. 
Suppose that a constant voltage difference $U=$ $V\left(L_{B}\right)-V\left(-L_{A}\right)$ is applied between the two ends of the system. Then, according to Eq. (A8),

$$
\int_{-L_{A}}^{L_{B}} d x E(x, t)=\int_{-L_{A}}^{L_{B}} d x \frac{\frac{j_{0}(t)}{q}+\sum_{i} D_{i} z_{i} \frac{\partial n_{i}}{\partial x}}{\frac{F}{R T} \sum_{i} D_{i} z_{i}^{2} n_{i}}=-U
$$

Thus the instantaneous value of the current density $j_{0}(t)$ is determined by the applied tension and by the instantaneous concentration fields $n_{i}(x, t)$ :

$$
\frac{j_{0}(t)}{q}=\frac{-\frac{F U}{R T}-\int_{-L_{A}}^{L_{B}} d x \sum_{i} D_{i} z_{i} \frac{\partial n_{i}}{\partial x}\left(\sum_{i} D_{i} z_{i}^{2} n_{i}\right)^{-1}}{\int_{-L_{A}}^{L_{B}} d x\left(\sum_{i} D_{i} z_{i}^{2} n_{i}\right)^{-1}} .
$$

At its turn, $j_{0}(t)$ determines through Eq. A8 the instantaneous electric field $E(x, t)$, that enters the evolution equations A4 A7.

One concludes that Eqs. A1, A2, (A4), A5, A6 , (A7) for the concentration profiles, A8 for the electric field, and A10 for the current density are all coupled in a highly nonlinear, intricate way. In our actual study (see Sec. II), we used several simplifications to make these equations more tractable.

\section{APPENDIX B: MORE NUMERICAL RESULTS}

Here we shall present additional results from our numerical simulations. We did not describe them in the main text, in order to make the essential results more transparent. However, we think they may put supplementary light on our conclusions, as well as on some controversial points of previously published results.

\section{Influence of finite-size effects on front's motion}

This section intends to strengthen the conclusion presented in the main text, namely that the finite-size effects do not influence the motion of the front as long as this one does not 'hit' the right border. This reinforce also the idea that the factors responsible of front's motion are strictly local.

In Fig. 9] we considered three system sizes and observed the long-time behavior: (i) for the smallest system the front hits the right border during the simulation time; (ii) for the intermediate system the front starts to 'feel' the border at the end of the simulations; (iii) in the the longest system, the front is unaffected by the borders during the simulation time.

Note however the very long time required to see such border effects even for the shortest system (about $10^{4}$

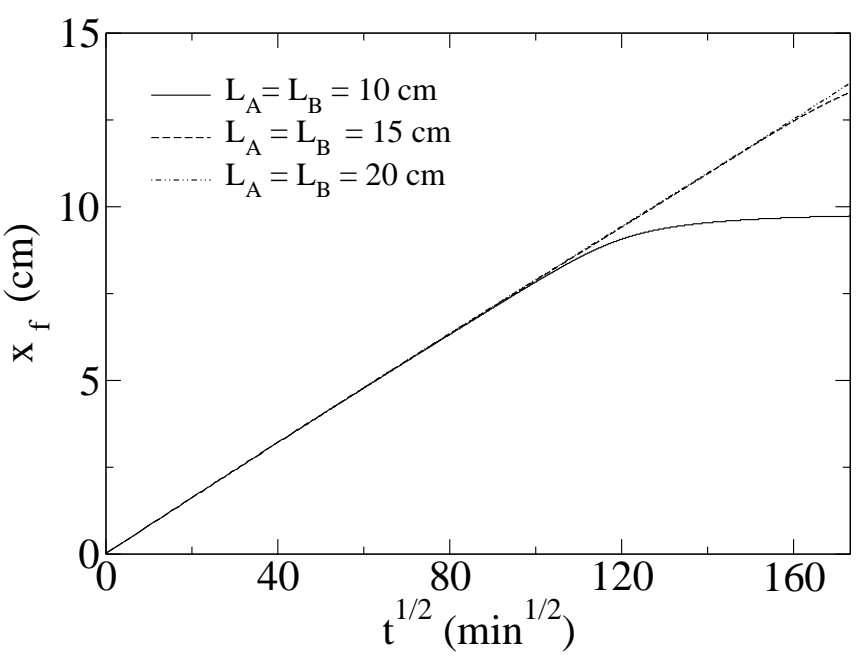

FIG. 9: Position of the reaction front as a function of the square root of time for different values of the system length. The values of the other parameters are: $a_{0}=10 \mathrm{M}, b_{0}=0.1$ $\mathrm{M}$, and applied electric field $U / L=0.5 \mathrm{~V} / \mathrm{m}$. The observation time is of 500 hours.

minutes for a system with $L_{B}$ of only $10 \mathrm{~cm}$ ). In most of the real experiments (for which both the system length is bigger - of at least a few tenths centimeters - and the observation time is usually smaller - a few days), as well as in most of our simulations, this slowing-down regime of the front is practically never attained.

\section{The current density $j_{0}$}

For a constant applied tension $U$ the current density $j_{0}$ is a function of time only; it is a global quantity, being determined by an integral over the entire system involving the instantaneous concentration fields. Consequently, $j_{0}(t)$ is sensitive to finite-size effects all along its evolution. The different regimes of $j_{0}(t)$ result essentially from the interplay between the evolving relative electric resistances of the depletion zone and that of the rest, 'unperturbed part' of the system.

The depletion zone is defined as the region where the concentrations of the ions are significantly smaller than their initial values. This region expands progressively, more or less rapidly (depending on the applied tension) around the moving reaction front. It can develop, of course, up to the size that is allowed by the borders of the system. It is a region of higher resistivity than the 'unperturbed' part of the system. There are two elements (with distinct temporal evolution) that determine the electric resistance of the depletion zone, namely (i) its spatial extent and (ii) its degree of depletion, i.e., the concentrations of the ions in the region. Note that the 
resistivity of the unperturbed part of the system is determined by its spatial extent, which equals $L$ minus the length of the depletion zone.

At the initial stages, the spatial extent of the depletion zone is small as compared to the rest of the system, and the concentrations of the ions are not extremely low yet. Therefore, its contribution to the electrical resistance of the column is negligible; during this stage, the resistance of the column is essentially constant and determined by the 'unperturbed' part of the system. Consequently, one obtains an initial regime of constant current density. This can be observed in Fig. 10 from which one also realizes that this is actually the regime that is accesible experimentally, and thus the only of interest to us.

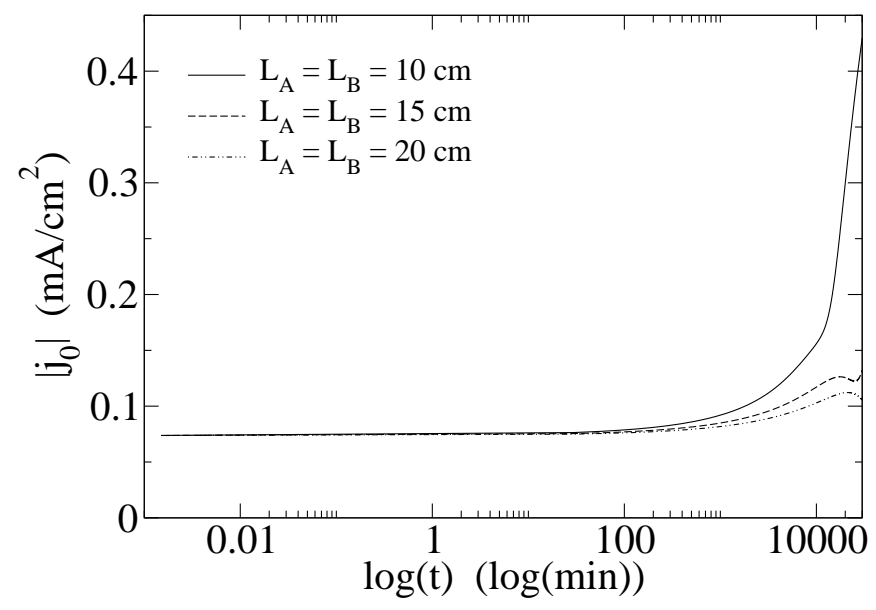

FIG. 10: The absolute value of the current density $\left|j_{0}\right|$ as a function of time (logarithmic scale) for various system sizes the same systems as those in Fig. 9] The values of the other parameters are: $a_{0}=10 \mathrm{M}, b_{0}=0.1 \mathrm{M}$, and applied electric field $U / L=0.5 \mathrm{~V} / \mathrm{m}$. The observation time is of 500 hours.

Although we shall not concentrate on them further (as not relevant for the typical experimental domains) let us briefly describe the other regimes that can be seen in Fig. [10] in the final part of the simulations.

(i) For the shortest system, the depletion zone cannot develop significantly before the front reaches the right border. Then the current passes directly from the constant-value regime to a rapid increase. When the front is in the vicinity of the border, the right-hand (with respect to the front) high-resistivity region of small concentration of the ions reduces progressively in size, while there is an advancement, behind the front, of a region of low-resistivity, high ion concentrations. This generates a decrease in the resistance of the system, and thus the observed increase in the current density.

(ii) For the intermediate and the longest system, the depletion region has more time to develop. At a certain time (shorter for the intermediate system), the electric resistivity of the depletion zone becomes comparable to that of the rest of the system. Then a transient regime starts - seen as an increase in $\left|j_{0}\right|$ for both systems. Finally, the increasing resistivity of the depletion zone becomes dominating in the system, causing a monotonous decrease in the current density $\left|j_{0}\right|$. However, the intermediate system does not 'have time' to develop this regime further: it hits the border and starts the corresponding increase in $\left|j_{0}\right|$, according to the mechanism sketched at point (i) above. A longer time simulation (not shown here) for the longer system shows a continuation of this regime, with $j_{0}(t) \sim 1 / \sqrt{t}$.

\section{The electric field}

The electric field is not constant along the system, as can be seen from Fig. [11 in contradiction with the fundamental assumption in 20]. This can be easily understood by inspection of Eq. (A8), taking into account that the concentration fields vary along the system. For a polarity of the externally applied tension $U$ that favors the reaction, the absolute value of the electric field increases rapidly in the vicinity of the moving front. This effect is due to the decrease in both the the concentrations of the reacting ions $A^{-}$and $B^{+}$(due to the reaction), and the concentrations of the background ions $A^{+}$and $B^{-}$ (because the polarity of the electric field pushes them away from the reaction zone). As a result, the sum in the denominator of the expression (A8) determining the electric field $\sum_{i} D_{i} z_{i}^{2} n_{i}$ becomes small in the vicinity of the reaction front, and thus the local field becomes very large.
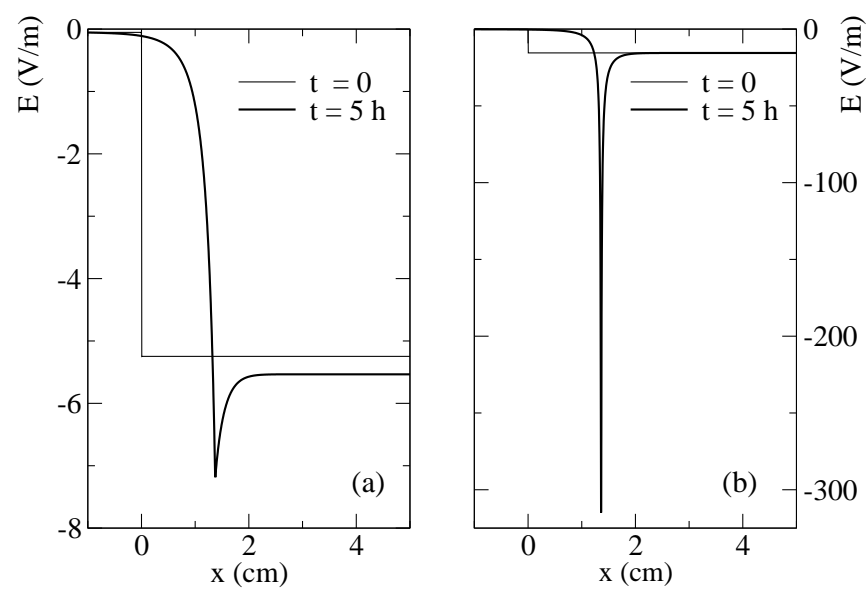

FIG. 11: Snapshots of the electric field $E(x, t)$ at two times and for two different values of the electric field $U / L$ applied to the system: (a) $U / L=5 \mathrm{~V} / \mathrm{m}$ (b) $U / L=15 \mathrm{~V} / \mathrm{m}$. The values of the other parameters are: $L_{A}=1 \mathrm{~cm}, L_{B}=20 \mathrm{~cm}$, $a_{0}=10 \mathrm{M}$, and $b_{0}=0.1 \mathrm{M}$. 
This rapid - both spatial and temporal, see Fig. 11increase in the electric field renders the numerical procedure unstable after some time. The other element that contributes to this instability is also connected with the small densities of the ions around the reaction zone: namely, from one integration step to another, the local concentration profiles may acquire unphysical negative values. These instabilities develop faster for larger applied tensions; their appearance can be delayed by decreasing both the spatial and temporal discretization steps. One is thus limited in the choice of the upper value of the applied tension $U$ by a 'reasonable' choice of the spatial and temporal discretization steps, that allow the study of the system for time intervals that are physically relevant. From a technical point of view, these instabilities are promptly notified by the value of the electric field at the reaction point, that acquires an unphysical temporal evolution at the moment of the onset of the instabilities.

The appearance of these numerical instabilities can be also postponed by: (i) giving up the infinite dissociation rate approximation, and allowing the $A$ and $B$ electrolytes to diffuse towards the reaction zone and to supply it with ions $A^{ \pm}$and $B^{ \pm}$through dissociation; (ii) taking into account the finiteness of the reaction rate $k$ between $A^{-}$and $B^{+}$, that leads to a slower decrease of the concentration of the reacting ions in the reaction zone. Note that in this case the reaction zone acquires a finite spatial extent (i.e., it is no longer reduced to a single point).

Finally, we should mention in connection with the electric field that, in real systems, this one has supplementary inhomogeneities related to the geometry of the system and, furthermore, the electric stability of the gel column may also reduced by electric effects at the walls of the container 31].

\section{The concentrations of the ions}

The concentration profiles $n_{i}(x, t)$ of the ions have a very complex evolution, that is determined by their diffusion coefficients, the initial concentrations, the length of the system, and the applied electric tension. A systematic presentation of all the corresponding effects would be tedious, and thus we shall present below only a few relevant elements.

For a given system (i.e., with fixed parameters), a qualitative typical temporal evolution of the concentration profiles corresponds to a reduction of the concentration of the ions in the vicinity of the moving reaction front, as well as to a progressive extension of this depletion zone (which, recall, is defined as the region where the concentrations of the ions are significantly smaller than their initial values); of course, as long as permitted by the boundaries, on which the concentrations of the ions are fixed. This is illustrated in Fig. 12

This depletion effect is more pronounced when the po-
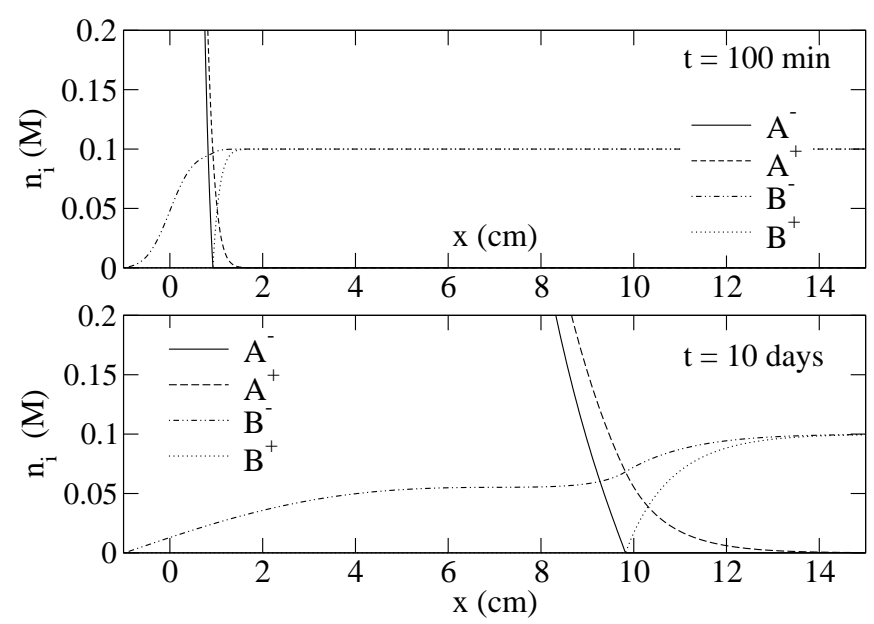

FIG. 12: Two snapshots of the concentration profiles of the ions in the vicinity of the reaction front at two different times. The values of the parameters of the system are: $L_{A}=1 \mathrm{~cm}$, $L_{B}=100 \mathrm{~cm}, a_{0}=10 \mathrm{M}$, and $b_{0}=0.1 \mathrm{M}$, and applied electric field $U / L=1 \mathrm{~V} / \mathrm{m}$.

larity of the applied tension is such that it favors the reaction than in the case of the opposite polarity. This can be easily understood: in an electric field that favors the reaction, not only the disappearance of the ions $A^{-}$ and $B^{+}$is favorized through reaction, but also the background ions $A^{+}$and $B^{-}$are pushed away from the reaction zone. Of course, in a field of reverse polarity, the nonreacting ions $A^{+}$and $B^{-}$are pushed towards each other, and the active ions $A^{-}$and $B^{+}$are pushed away from each other (and thus their consumption through reaction is reduced; this leads, in the end, to an extinction of the reaction).

Finally, for a polarity of the applied tension that is favorable to the reaction, the expansion of the depletion zone is more rapid and pronounced for larger tensions. This determines the local increase of the electric field, and, in the long time, the numerical instability of the integration procedure (as discussed in the previous subsection).

\section{The concentration of the reaction product $C$}

For the regime of constant $j_{0}$, as discussed in the main text, the concentration of the reaction product $c(x)$ increases linearly with $x$ with a slope proportional to the fieldless value $c_{0}$, as illustrated by Fig. 13 .

This initial behavior of the production of $C$ is determined by and lasts as long as the initial regime of constancy of the current density $j_{0}$, and therefore it is the only regime on which we focus in our study. However, as discussed above in the corresponding subsection, the 


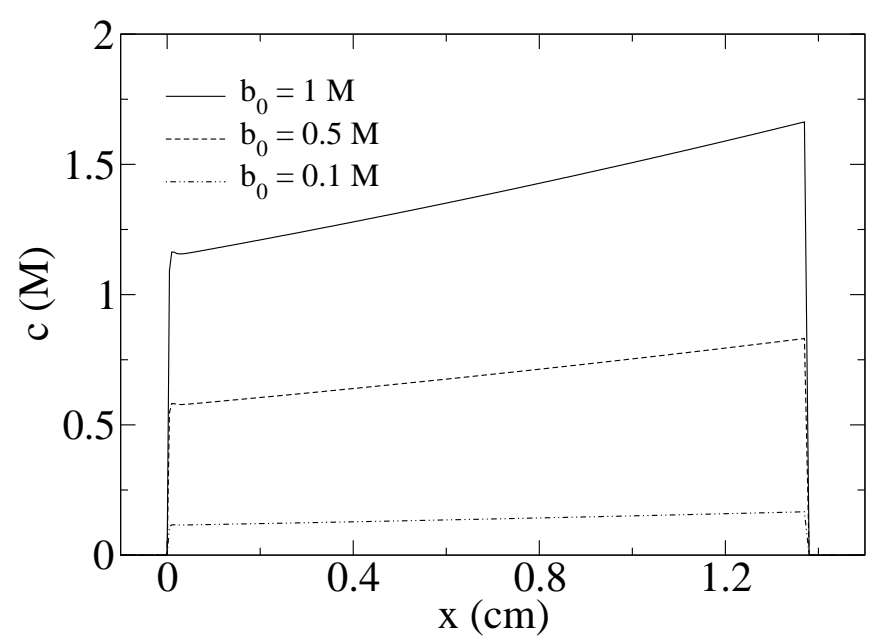

FIG. 13: The density of the reaction product $C$ left behind the reaction front for different values of the concentration $b_{0}$. The observation time is $t=5$ hours. The values of the parameters of the system are: $L_{A}=1 \mathrm{~cm}, L_{B}=20 \mathrm{~cm}$, fixed ratio $a_{0} / b_{0}=100$, and the applied electric field $U / L=5 \mathrm{~V} / \mathrm{m}$. current density $j_{0}$ may develop other regimes (if allowed by the size of the system). Then, of course, these regimes will be reflected by the production of $C$. In particular, the transient increase (if any) in $\left|j_{0}\right|$ between the initial regime of constancy and the regime $j_{0} \sim 1 / \sqrt{t}$ is reflected by a transient increase in the production of $C$ (a "bump" in the profile $c(x))$. The regime $j_{0} \sim 1 / \sqrt{t}$ itself leads to a constant deposition of $C$ in the wake of the front; the plateau value of $c$ varies with $a_{0}$ and $b_{0}$ (much in the same way as $c_{0}$ ), but is rather insensitive to the applied tension $U$. Finally, when the reaction front 'hits' the right border, there is a great increase in the production of $C$ at the border.

\section{ACKNOWLEDGMENTS}

We thank I. Lagzi for very useful discussions. This research has been partly supported by the Swiss National Science Foundation and by the Hungarian Academy of Sciences (Grants No. OTKA T043734 and TS 044839).
[1] For a review, see M. C. Cross and P. C. Hohenberg, Rev. Mod. Phys. 65, 851 (1994).

[2] R. E. Liesegang, Naturwiss. Wochenschr. 11, 353 (1896).

[3] H. K. Henisch, Periodic Precipitation (Pergamon, New York, 1991).

[4] It should be noted that, using special initial conditions, precipitation patterns may also form without the presence of reaction zones [see for a recent example S. C. Müller and J. Ross, J. Phys. Chem. A107, 7997 (2003)]. In a usual Liesegang experiment, however, the reaction front is easily detectable and the emergence of the precipitation bands is strongly correlated with the position of the front.

[5] See, e.g., Z. Rácz, Physica A 274, 50 (1999).

[6] T. Antal, M. Droz, J. Magnin, Z. Rácz, and M. Zrinyi, J. Chem. Phys. 109, 9479 (1998).

[7] J. Magnin, Ph.D. Thesis, University of Geneva (2000).

[8] T. Antal, M. Droz, J. Magnin, and Z. Rácz, Phys. Rev. Lett. 83, 2880 (1999).

[9] T. Antal, M. Droz, J. Magnin, A. Pekalski, and Z. Rácz, J. Chem. Phys. 114, 3770 (2001).

[10] This is a delicate point. Indeed, one could argue for example that the subsequent precipitation of $C$ would change the mobility of the reagents. There are no experimental indications on the relevance of these effects, and thus on the correctness of our assumption. It can receive some aposteriori justification - through the fact that it leads to quantitatively correct final results.

[11] J. D. Gunton, M. San Miguel, and P. S. Sahini, in Phase Transitions and Critical Phenomena, Vol. 8, The Dynamics of First-order Transitions, Eds. C. Domb and J. L. Lebowitz (Academic Press, New York, 1983).
[12] J. W. Cahn and J. E. Hilliard, J. Chem. Phys. 28, 258 (1958).

[13] J. W. Cahn, Acta Metall. 9, 795 (1961).

[14] P. C. Hohenberg and B. I. Halperin, Rev. Mod. Phys. 49, 435 (1977).

[15] L. Gálfi and Z. Rácz, Phys. Rev. A 38, 3151 (1988).

[16] T. Unger and Z. Rácz, Phys. Rev. E 61, 3583 (2000).

[17] P. Happel, R. E. Liesegag, and O. Mastbaum, Kolloid Z. 48, 80 (1929); ibid., 48, 252 (1929).

[18] B. Kisch, Kolloid Z. 49, 154 (1929).

[19] P. Ortoleva in Theoretical Chemistry, Vol. IV, edited by H. Eyring (Academic Press, New York, 1978).

[20] R. Feeney, S. L. Schmidt, P. Strickholm, J. Chadam, and P. Ortoleva, J. Chem. Phys. 78, 1293 (1983).

[21] A. H. Sharbaugh III and A. H. Sharbaugh Jr., J. Chem. Educ. 66, 589 (1989).

[22] I. Das, A. Pushkarna, and A. Bhattacharjee, J. Phys. Chem. 94, 8968 (1990).

[23] I. Das, A. Pushkarna, and A. Bhattacharjee, J. Phys. Chem. 95, 3866 (1991).

[24] I. Das, A. Pushkarna, and S. Chand, J. Coll. Int. Sci. 150, 178 (1992).

[25] D. S. Chernavskii, A. A. Polezhaev, and S. C. Müller, Physica D 54, 160 (1991).

[26] A. A. Polezhaev and S. C. Müller, Chaos 4, 631 (1994).

[27] R. Sultan and R. Halabieh, Chem. Phys. Lett. 332, 331 (2000).

[28] M. Al-Ghoul and R. Sultan, J. Phys. Chem. A 107, 1095 (2003).

[29] I. Lagzi, Phys. Chem. Chem. Phys. 4, 1268 (2002).

[30] I. Lagzi and F. Izsák, Phys. Chem. Chem. Phys. 5, 4144 (2003). 
31] I. Lagzi, private communication.

[32] Thus the problem of the dependence of the width of the reaction front on the applied electric field is not approached here, but left for further studies.

[33] See, e.g., I. Rubinstein, Electrodiffusion of Ions (SIAM, Philadelphia, 1990); J. Koryta, J. Dvorak, and L. Kavan, Principles of Electrochemistry (John Wiley \& Sons, New York, 1993).

[34] T. Unger, Master Thesis, Eötvös University, Budapest,
Hungary (1999).

[35] $U=$ constant is the most common experimental situation. But one can equally well consider experimental situations for which the current density $j_{0}$ is maintained constant. However, as discussed in some more detail in Appendix B2, for the experimentally relevant observation times there is no significant difference between these two situations. 